\title{
Probing high-pressure properties of single-wall carbon nanotubes through fullerene encapsulation
}

\author{
Ch. Caillier, D. Machon, and A. San-Miguel \\ Université de Lyon, F-69000, France \\ and Laboratoire PMCN, CNRS, UMR 5586, Université Lyon 1, F-69622 Villeurbanne, France \\ R. Arenal \\ Laboratoire d'Étude des Microstructures, ONERA-CNRS (UMR 104), 92322 Châtillon, France \\ and Materials Sciences Division, Argonne National Laboratory, Argonne, Illinois 60439, USA \\ G. Montagnac and H. Cardon \\ Université de Lyon, F-69000, France \\ and Laboratoire des Sciences de la Terre, ENS-Lyon, CNRS, Université Lyon 1, F-69364 Lyon, France \\ M. Kalbac, M. Zukalova, and L. Kavan \\ J. Heyrovsky Institute of Physical Chemistry, v.v.i. Academy Sciences of the Czech Republic, Dolejskova 3, Prague 182238 , \\ Czech Republic
}

(Received 19 December 2007; published 18 March 2008)

\begin{abstract}
The high pressure behavior of bundled $1.35 \pm 0.1 \mathrm{~nm}$ diameter single wall carbon nanotubes (SWNT) filled with $\mathrm{C}_{70}$ fullerenes (usually called peapods) has been investigated by Raman spectroscopy and compared with the corresponding behavior of the nonfilled SWNT. We show experimentally that two reversible pressureinduced transitions take place in the compressed bundle SWNT. The first transition, in the 2-2.5 GPa range, is in good correspondence with predictions of the thermodynamic instability of the nanotube circular cross section for the studied tube diameter. An interaction between the fullerenes and the tube walls is then observed at about $3.5 \mathrm{GPa}$, which evidences a progressive deformation of the tube cross section. The second transition takes place at pressures between 10 and $30 \mathrm{GPa}$, and is evidenced by two effects by a strong frequency downshift of the Raman transverse modes and the concomitant disappearance of the fullerenes Raman modes in peapods. The pressure at which the second transition takes place is strongly dependent on the nature of the pressure transmitting medium. We also report irreversible effects at high pressure as the shortening of the tubes, the formation of nanostructures and the disappearance of the $\mathrm{C}_{70}$ Raman signal in some cases. Transmission electron microscopy studies are also reported supporting these transformations.
\end{abstract}

DOI: 10.1103/PhysRevB.77.125418

PACS number(s): 78.30.Na, 61.46.Fg, 62.50.-p, 78.67.Ch

\section{INTRODUCTION}

The exceptional mechanical properties of carbon nanotubes are generating a huge scientific activity. The high value of their Young modulus or their high resilience is an example of those properties that find applications in the development of fibers or nanocomposite materials among others. In spite of the extensive investigation of their mechanical properties, important discrepancies between experiments and theory persist as well as between models themselves. In the case of single wall carbon nanotubes, to which we will restrict our discussion here, both theoretical works ${ }^{1-8}$ and experiments $^{9-13}$ agree in pointing out the presence of a pressure-induced transition. Calculations propose that this transition involves a modification of the nanotube cross section from circular to oval, polygonized, or flattened, associated in some cases to an important volume collapse. Experimentally, the discrimination between these different possibilities has not been carried out. In addition, some calculations predict different types of modifications depending on the tube size or chirality. ${ }^{14}$

Most calculations agree on transition pressures scaling as $1 / d^{3}$, where $d$ is the nanotube diameter, ${ }^{3,4,7}$ as for the classical elastic theory of rings. ${ }^{15}$ For the most commonly studied tubes having a size distribution centered around $1.4 \mathrm{~nm}$, the predicted transitions range in the 1-2 GPa pressure domain. These predictions are corroborated by the observed modifications of the Raman signal at pressures between 1 and $3 \mathrm{GPa}$ in bundled ${ }^{9,11,16}$ and individualized nanotubes. ${ }^{17,18} \mathrm{On}$ the contrary, strong changes in the Raman ${ }^{10}$ or x-ray diffraction ${ }^{19}$ signals point out transitions in the $10 \mathrm{GPa}$ domain for tubes of similar sizes. We show here that bundled nanotubes of diameter $1.35 \pm 0.1 \mathrm{~nm}$ undergo two transitions in two distinct pressure domains. In peapods, where the encapsulated $\mathrm{C}_{70}$ fullerenes served as internal probes, the two transitions are also observed. The fullerene signal allowed to discriminate between some of the theoretical predictions for the first transition and provides some insight into the nature of the second one.

Dynamics of materials under high pressure can be studied with different techniques including neutron ${ }^{20}$ or x-ray inelastic scattering. ${ }^{21}$ In the case of carbon nanotubes, optical resonance linked to their one-dimensional character makes Raman spectroscopy an extremely powerful technique to study their evolution. ${ }^{22}$ Both the attenuation of the radial breathing modes $^{9}$ (RBMs) and changes in the pressure derivative of the tangential modes ${ }^{10}$ (TM) have been invoked as indicators of pressure-induced transformations..$^{13}$ In some cases, a change of sign of the pressure derivative of the TM frequencies has been observed. ${ }^{10,18}$ For individualized nanotubes, the simul- 

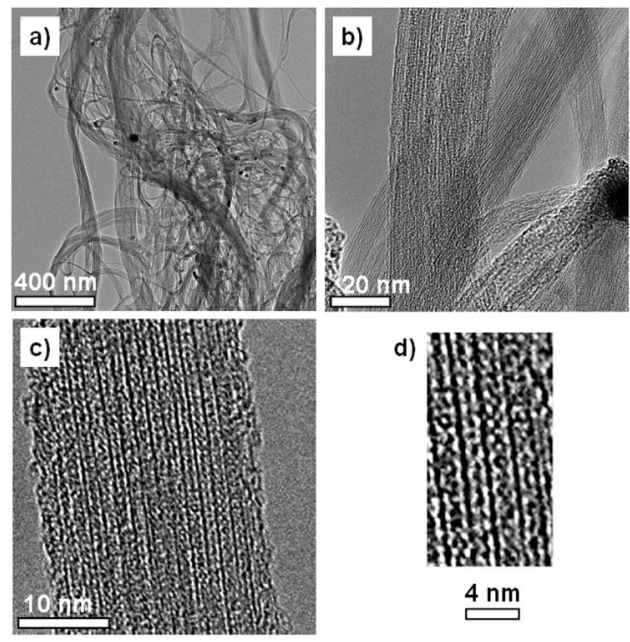

FIG. 1. Transmission electron microscopy images of the initial sample of peapods with different enlarging. It can be seen in (a) and (b) that the tubes are organized in bundles. From (c) and (d), we estimate the diameter of these tubes, close to $1.5 \mathrm{~nm}$, and their filling factor, higher than $80 \%$.

taneous attenuation of RBM and TM has been observed. ${ }^{17}$ In the case of bundled tubes, the loss of resonance of the RBM has been proposed to explain its attenuation. ${ }^{23}$ This evidences one aspect of the difficulty in confronting the various experimental observations and theoretical predictions. In order to gain some insight in this complex panorama, the introduction of an internal probe inside the nanotubes is the strategy chosen in this paper. Fullerenes are good candidates for such an internal probe as they can easily fill nanotubes, their Raman signal is well defined and intense enough, and there exist previous high pressure studies. The $\mathrm{C}_{70}$ fullerene possesses an advantage with respect to $\mathrm{C}_{60}$ since the polymerization of the $\mathrm{C}_{70}$ oval molecule inside carbon tubes of rather small diameters is less favorable, as the preferred polymerization orientation is zig zag. ${ }^{24,25}$ In the present study, indeed, we have not found any indication of pressureinduced polymerization of $\mathrm{C}_{70}$ inside the nanotubes.

Several pressure studies have been carried out on $\mathrm{C}_{60}$ peapods, ${ }^{26-28}$ but less on $\mathrm{C}_{70}$ peapods. ${ }^{24,29}$ To our knowledge, there has been only one Raman study on $\mathrm{C}_{70}$ peapods under pressure. ${ }^{29}$ In that work, the behavior of $\mathrm{C}_{60}$ and $\mathrm{C}_{70}$ peapods was investigated by Raman spectroscopy from 0 to $9 \mathrm{GPa}$. It revealed that the effective pressure acting on the $\mathrm{C}_{60}$ fullerenes is screened by the tube. For $\mathrm{C}_{70}$ peapods, the evolution of three fullerene Raman modes led the authors to conclude to a transition at around $2 \mathrm{GPa}$. This transition was interpreted as the switching of the oval $\mathrm{C}_{70}$ molecules from standing to lying position inside the tubes.

\section{EXPERIMENTS}

Bundled $\mathrm{C}_{70} @ \mathrm{SWNT}$ peapods (where SWNT denotes single wall nanotube) were prepared as buckypaper samples following a method described elsewhere. ${ }^{30,31}$ By electron microscopy and $\mathrm{x}$-ray diffraction, we estimated the filling factor to be at least $80 \%$, with a very low rate of nonendohedral $\mathrm{C}_{70}$ fullerenes (see Fig. 1). The diameter of the tubes ranges from 1.25 to $1.47 \mathrm{~nm}$, as estimated by Raman spectroscopy and X-ray diffraction. ${ }^{30}$ A sample of empty closed nanotubes used to produce these peapods was also studied as a reference.

Transmission electron microscopy (TEM) studies were performed on a FEI Tecnai F20 FEG TEM/STEM microscope operating at $200 \mathrm{kV}$. High resolution TEM images [Figs. 1(c) and 1(d)] confirm the former values for the tube diameters. In fact, it is well established ${ }^{32,33}$ that in this kind of image, the fringes observed correspond to the (11) planes of the two-dimensional triangular network formed by the nanotubes in the bundle arrangement in the longitudinal projection. In such case, these images are not directly related to the individual nanotube atomic structure, but to that of the bundle network. Thus, measuring the distance between two of those fringes, the diameter dispersion of the nanotube can be deduced. ${ }^{33}$ For the observed bundle [Fig. 1(d)], we found a diameter close to $1.5 \mathrm{~nm}$, which is in agreement with the $1.25-1.47 \mathrm{~nm}$ range. It has been shown recently that the critical diameter for the switch between standing and lying orientations of the $\mathrm{C}_{70}$ molecules inside a single wall carbon nanotube (SWNT) lies between 1.42 and $1.44 \mathrm{~nm} .{ }^{34}$ In our sample, we thus expect that, even if some $\mathrm{C}_{70}$ molecules can be found in standing configuration as shown by $\mathrm{x}$-ray diffraction, ${ }^{30}$ most of the $\mathrm{C}_{70}$ molecules are in lying orientation.

High pressure experiments were carried out by using a diamond anvil cell with a metal gasket. The hole of the gasket serving as the compression chamber was set to $125 \mu \mathrm{m}$ in diameter and $35 \mu \mathrm{m}$ in thickness. This chamber was filled with a small piece of buckypaper, a ruby chip for pressure calibration, and the pressure transmitting medium (PTM). Three PTMs were used: paraffin oil, a 4:1 methanol:ethanol mixture, and argon, referred in the following as oil, alcohol, and argon, respectively. Raman spectra were measured in situ in backscattering geometry on a Jobin Yvon Labram HR800 spectrometer, with a spectral resolution of about $0.5 \mathrm{~cm}^{-1}$. The laser wavelength was $514.5 \mathrm{~nm}$, which optimizes the resonant Raman signal of both nanotubes and fullerenes. $^{29}$

We could, thus, observe separately the RBMs [Fig. 2(a)] and TMs or $G$ band [Fig. 2(c)] of the tubes around $160-200 \mathrm{~cm}^{-1}$ and $1400-1600 \mathrm{~cm}^{-1}$, respectively, as well as the spectral region between the RBM and the $D$ band $\left(350-1280 \mathrm{~cm}^{-1}\right)$, containing many weak modes from the $\mathrm{C}_{70}$ fullerenes and some nanotube intermediate modes [Fig. 2(b) in oil and Fig. 3 in alcohol]. The $\mathrm{C}_{70}$ modes are clearly discernible from the SWNT ones, which have a full width at half maximum (FWHM) significantly larger because of the nanotube diameter distribution. A comparison with the spectra of the empty nanotubes at different pressures allows to distinguish the $\mathrm{C}_{70}$ modes from the other components (nanotubes or PTM bands). The positions of the $\mathrm{C}_{70}$ and nanotube modes at ambient pressure are shown as tick marks in the lower plot of Figs. 2(b) and 3. The spectra were recorded at different pressures up to the disappearance of most of the fullerene modes in oil (some modes remained at about $700 \mathrm{~cm}^{-1}$ ) and of all the modes in alcohol. The spectra recorded with argon as PTM are not shown as they are very similar to those of the alcohol case (see below). 


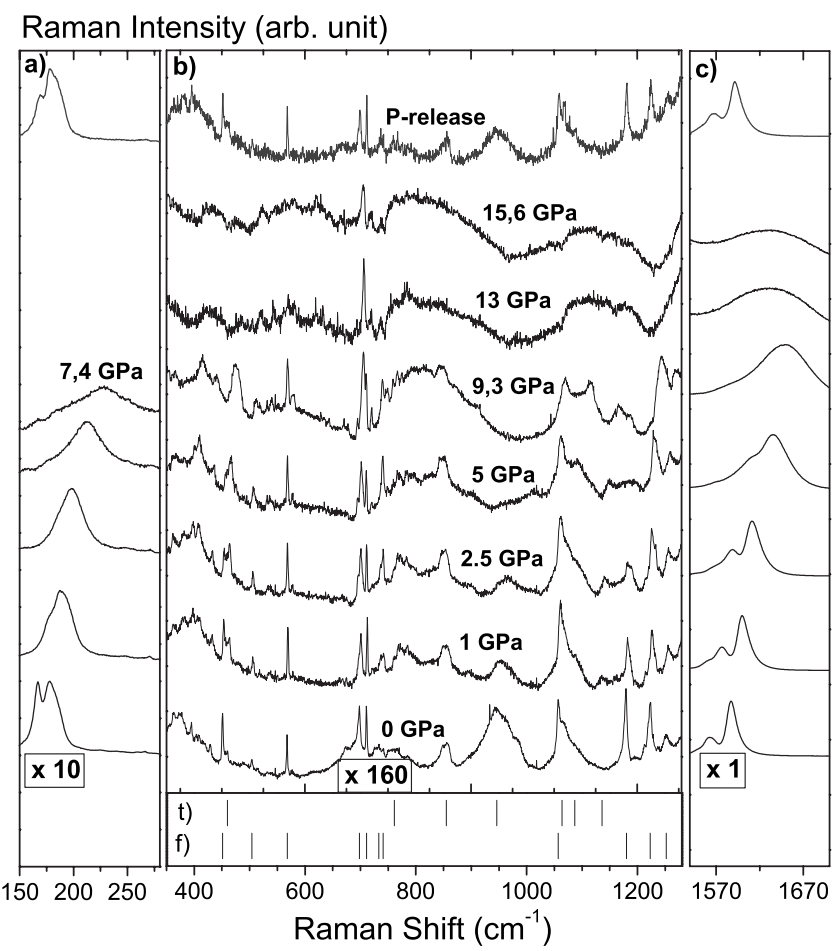

FIG. 2. Pressure evolution of the Raman spectra of $\mathrm{C}_{70}$ peapods when paraffin oil is used as pressure transmitting medium. (a) RBM signal up to its attenuation. (b) Spectral region between 350 and $1280 \mathrm{~cm}^{-1}$ dominated by the $\mathrm{C}_{70}$ modes. The lower central panel shows the peak positions corresponding to the contribution to the signal of (t) nanotubes and (f) $\mathrm{C}_{70}$ fullerenes at ambient pressure. (c) TM signal. The upper spectra shown in the three panels were measured after pressure release. The boxes under the lower spectra give the magnification of the different spectra at ambient pressure.

\section{RESULTS AND DISCUSSION}

\section{A. Low pressure transition}

A regular upshift of the RBM frequencies of about $6 \mathrm{~cm}^{-1}$ is observed up to their disappearance at around $8 \mathrm{GPa}$ in oil and at $14 \mathrm{GPa}$ in alcohol and argon. This upshift value is in the lower mean of former observations on single-walled carbon nanotubes ${ }^{9,13,17,35}$ and is consistent with values obtained on double-walled carbon nanotubes. ${ }^{36}$ In Fig. 4, the linewidth of the RBM is plotted versus the pressure for peapods in oil, alcohol, and argon, and empty nanotubes in oil. We observe the onset of a progressive increase of this linewidth between 2 and $2.5 \mathrm{GPa}$ in all cases, which is associated with the onset of the progressive attenuation of the RBM signal. Within our precision, this critical pressure is independent of the filling of the tubes as well as the choice of PTM. The independence from the endohedral filling of the value of this transition pressure strongly suggests the weak character of the fullerene-tube interaction from ambient pressure to $2 \mathrm{GPa}$. This is also supported by the weak pressure dependence of the Raman fullerene modes in this pressure domain as it will be discussed later. Contrarily to the transition pressure value, the broadening rate of the RBM after the transition strongly depends on the filling of the tubes, and slightly on the choice of PTM (see Fig. 4). The viscosity of the PTM,

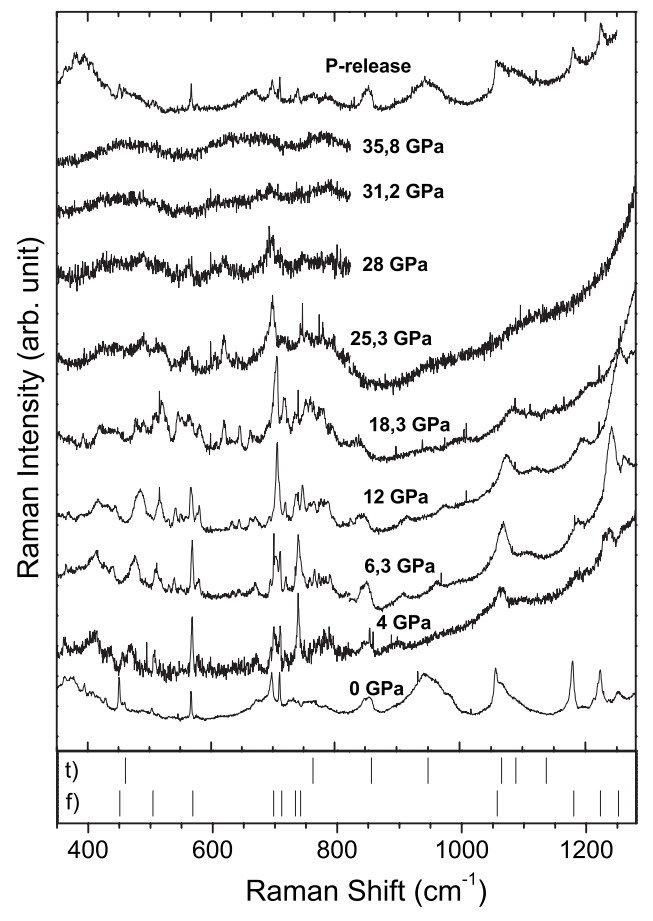

FIG. 3. Pressure evolution of the Raman spectra between 350 and $1280 \mathrm{~cm}^{-1}$ of the $C_{70}$ peapods when a $4: 1$ methanol:ethanol mixture is used as pressure transmitting medium. As in Fig. 2, the lower plot shows the peak positions corresponding to the contribution to the signal of $(\mathrm{t})$ nanotubes and (f) $\mathrm{C}_{70}$ fullerenes at ambient pressure.

or steric arguments having consequences on the modulation of the pressure-induced deformations, may explain these differences.

In Fig. 5(a) [Fig. 5(b)] we show the pressure evolution of the position of $\mathrm{C}_{70}$ Raman modes when oil (alcohol and argon) is used as PTM. It is worth noting that in this figure, as in Fig. 4, the results are exactly the same in alcohol and argon, which justifies why both results are presented together in the same graph. At $3.5 \mathrm{GPa}$, we observe in Fig. 5(a) a strong variation of three mode intensities (450, 740, and $1180 \mathrm{~cm}^{-1}$ ) and a clear change in the pressure derivative frequency of three other fullerene modes. Except for the modes between 565 and $745 \mathrm{~cm}^{-1}$, the frequencies of which are quite insensitive to pressure, most of the slopes change from less than $1 \mathrm{~cm}^{-1} / \mathrm{GPa}$ to several $\mathrm{cm}^{-1} / \mathrm{GPa}$, as shown in Table I. In the case of argon and ethanol as PTM [Fig. $5(\mathrm{~b})]$ this transition cannot be observed because the starting pressure was higher, due to the cell loading protocol. Nevertheless, according to the linear fits obtained from Fig. 5(a), the slopes are perfectly consistent with the values measured after the transition at $3.5 \mathrm{GPa}$. As shown in Table I, the posttransition values are also in good agreement with the ones found when compressing pristine $\mathrm{C}_{70}$ crystals, ${ }^{37}$ where all fullerenes lie in layers. All these observations are a sign of the onset of the interaction between the tubes and the fullerenes or/and between the fullerenes themselves. We note that the observed change in the pressure derivative of the $\mathrm{C}_{70}$ frequencies is of opposite sign to the ones observed in Ref. 29. The origin of such a discrepancy can be due to the fact 


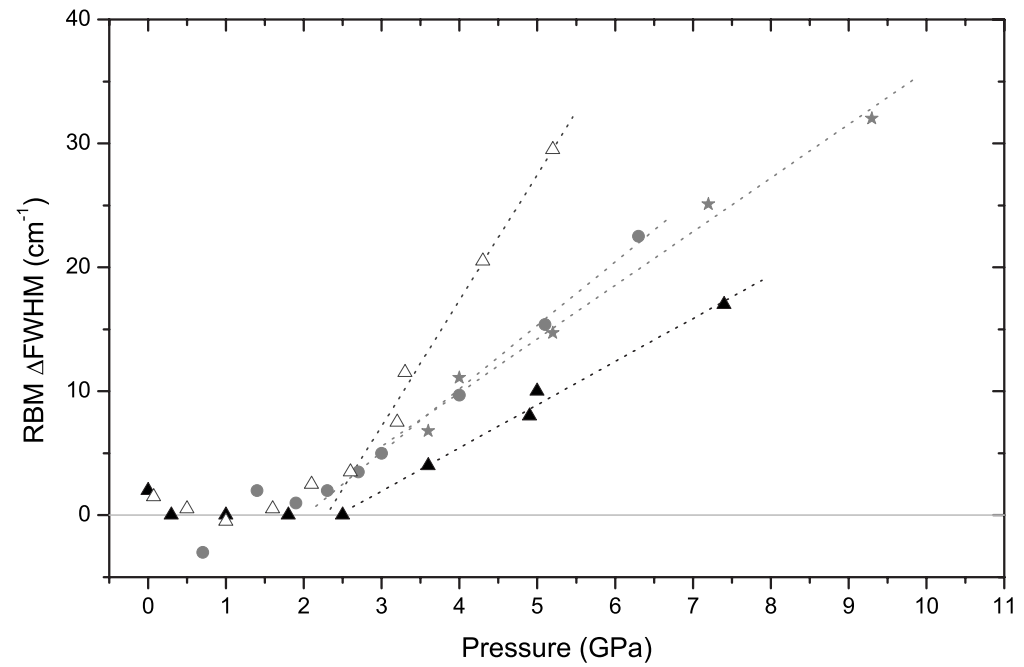

FIG. 4. Pressure variation of the RBM linewidth of the $\mathrm{C}_{70}$ peapods in oil (filled triangles), alcohol (filled circles), and argon (filled stars), and empty nanotubes in oil (hollow triangles). Dashed lines are linear fits after the transition.

that the very weak signal of the fullerenes observed in that work coincides with the signal of the PTM: a 4:1 methanol:ethanol mixture, which evolves under pressure as shown in Ref. 38.

The pressure values of $2-2.5 \mathrm{GPa}$, at which we observe the change of evolution on the RBM, are in good agreement with the theoretically predicted pressure of transition for our tube size. These predictions involve nanotube cross-section modifications, $1,2,4-7$ in agreement with other experimental observations. ${ }^{9,11,16,18}$ In addition, our results show that (i) the cross-section modification should be compatible with the presence of $\mathrm{C}_{70}$ fullerene inside the tubes, (ii) the transition does not give rise to an immediate enhancement of the fullerene-tube or fullerene-fullerene interaction, which takes place at higher pressures, and (iii) both the fullerene modes and the FWHM of the RBM show a monotonous behavior with pressure from the observed transition pressures. All these constraints exclude nanotube cross-section changes involving an important volume reduction, as, for instance, the flattening of the tubes. They also let us envisage a progressive modification of the nanotube cross section from the transition. Possible nanotube shapes compatible with such constraints and corresponding to several predictions, like ovalization and polygonization, are shown in Figs. 6(b) and 6(e).

\section{B. High pressure transition}

In Fig. 7 is shown the frequency of the TM maximum intensity vs the pressure for peapods in oil, alcohol, and argon. We see that the pressure derivative frequency $\partial \omega_{T M} / \partial P$ is, first, roughly constant with a value of about $10 \mathrm{~cm}^{-1} / \mathrm{GPa}$, which is in the higher mean of former observations on single-walled carbon nanotubes ${ }^{9,13,17}$ and higher

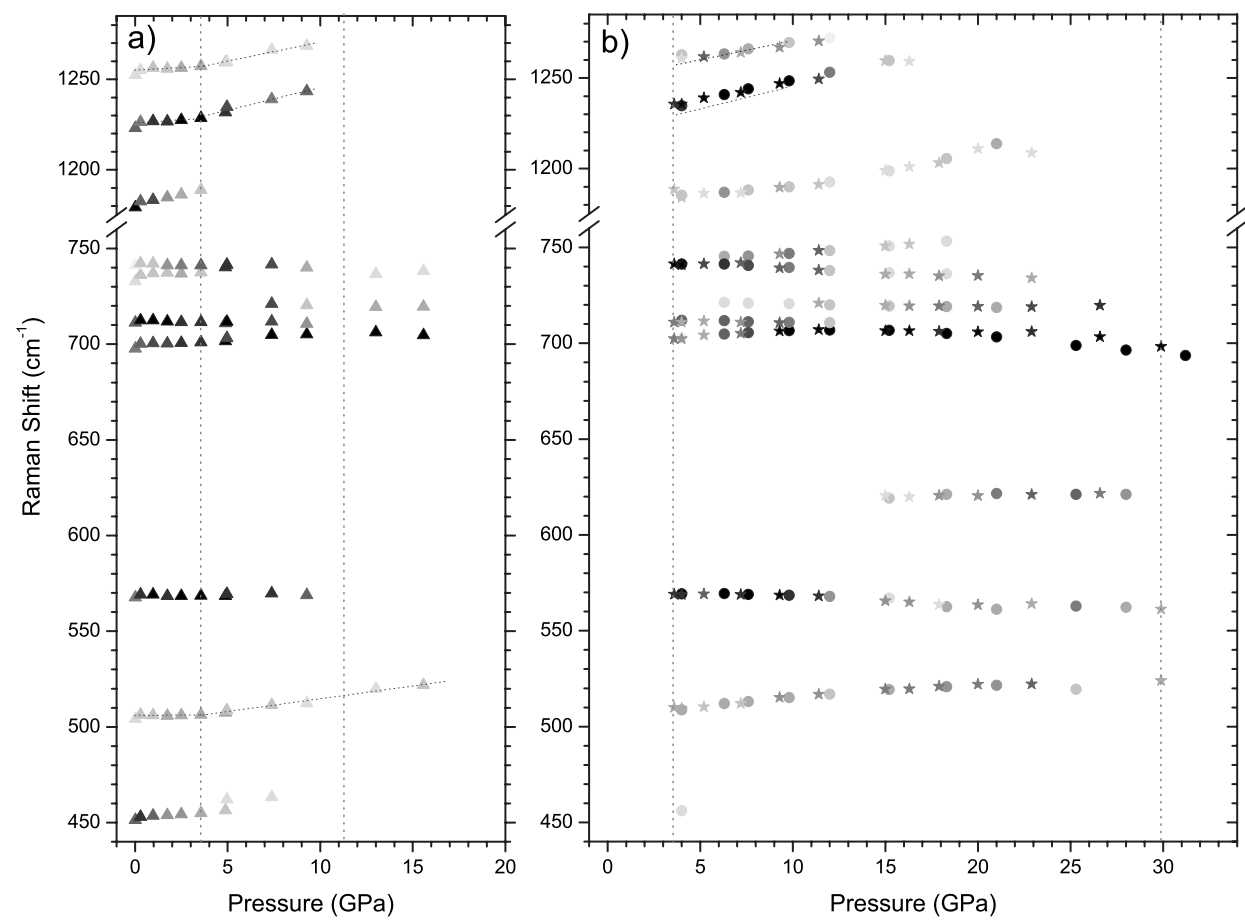

FIG. 5. Pressure evolution of the Raman mode frequencies for the encapsulated $\mathrm{C}_{70}$ fullerenes with (a) oil or (b) alcohol (circles) and argon (stars) as pressure transmitting medium. The mode intensities are normalized to the highest of each pressure. The obtained relative intensities are displayed in gray level. The vertical dashed lines mark the observed transitions (see text). Linear fits are included in (a) for modes having an important change in their pressure slopes upon the first transition. These fits are kept in (b) for comparison. 
TABLE I. Frequencies of the different $\mathrm{C}_{70}$ Raman modes $\left(\omega_{0}\right)$ and their pressure derivative $(d \omega / d P)$ in our peapod sample when the PTM is oil [see Fig. 5(a)] compared to the case of pristine $\mathrm{C}_{70}$ (Ref. 37). The mode frequencies at ambient pressure, $\omega_{0}$, obtained in this work (peapod) and in Ref. 37 (pristine) are shown in the first two columns. In the next three columns the measured pressure derivatives of these modes are given, $d \omega / d P$, before the transition at 3.5 GPa $\left(P<P_{t}\right)$, after the transition $\left(P>P_{t}\right)$ and as obtained in Ref. 37 (pristine), respectively. In the cases where a comparison is possible, we observe that the pressure derivative frequencies obtained after the transition and in pristine $\mathrm{C}_{70}$ compare better.

\begin{tabular}{lccccc}
\hline \hline \multicolumn{2}{c}{$\omega_{0}\left(\mathrm{~cm}^{-1}\right)$} & & \multicolumn{3}{c}{$d \omega / d P\left(\mathrm{~cm}^{-1} \mathrm{GPa}^{-1}\right)$} \\
\cline { 1 - 3 } \cline { 4 - 6 } Peapod & Pristine $^{\mathrm{a}}$ & & $P<P_{t}$ & $P>P_{t}$ & Pristine $^{\mathrm{a}}$ \\
\hline 451 & & 0.6 & 2.1 & \\
504 & & & 0.0 & 1.1 & \\
568 & 564 & & 0.0 & 0.0 & -0.06 \\
698 & & & 0.4 & 0.4 & \\
711 & & & -0.1 & -0.1 & \\
741 & 1065 & & 0.4 & 1.3 & 1.1 \\
1057 & 1182 & & 2.0 & Disapp. & $4 /-10.3$ \\
1179 & 1224 & & 0.6 & 2.5 & 3.2 \\
1223 & & 0.6 & 2.1 & \\
1252 & & & 0.6 & -0.2 & 0.12 \\
\hline \hline
\end{tabular}

${ }^{\text {aReference } 37 .}$

than values obtained on double-walled carbon nanotubes. ${ }^{36}$ The shift of the TMs under pressure can be related to the Gruneisen parameters and the applied mechanical strain ${ }^{39}$ as is also observed in electrochemical doping effects. ${ }^{40}$ An important frequency downshift is then observed in the 10-35 GPa range, depending on the experiment: $11 \pm 2 \mathrm{GPa}$ for peapods in oil, $14 \pm 1 \mathrm{GPa}$ for nonfilled tubes in oil, and $30 \pm 2 \mathrm{GPa}$ for peapods in alcohol or argon. No clear frequency downshift is observed for nonfilled tubes in alcohol or argon up to $43 \mathrm{GPa}$. On pressure release, a strong hysteresis is observed. The initial TM shape and frequency are recovered only at ambient pressure. For peapods, the key point is the simultaneity between this frequency downshift and the disappearance of most of the $C_{70}$ modes (Fig. 5),

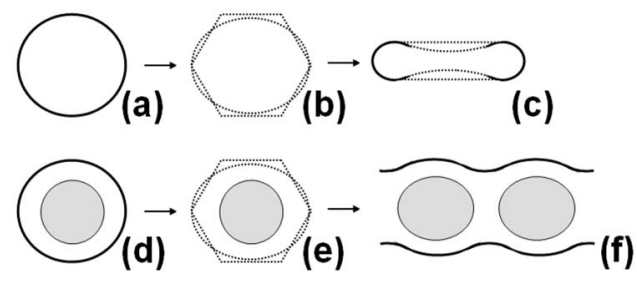

FIG. 6. Proposed pressure evolution of the nanotube shape in nanotube [from (a) to (c)] and peapods [from (d) to (f)]. Arrows correspond to the two observable transitions in each system at low pressure $[(a) \rightarrow(b)$ and $(\mathrm{d}) \rightarrow(\mathrm{e})]$ and at high pressure $[(\mathrm{b}) \rightarrow(\mathrm{c})$ and (e) $\rightarrow$ (f)]. (a)-(e) show the proposed cross sections: [(a) and (d)] circular, [(b) and (e)] elliptical or polygonized, and (c) racetrack or peanut type. In (f) is shown a longitudinal cut of the peapod after the second transition. See text for discussion.

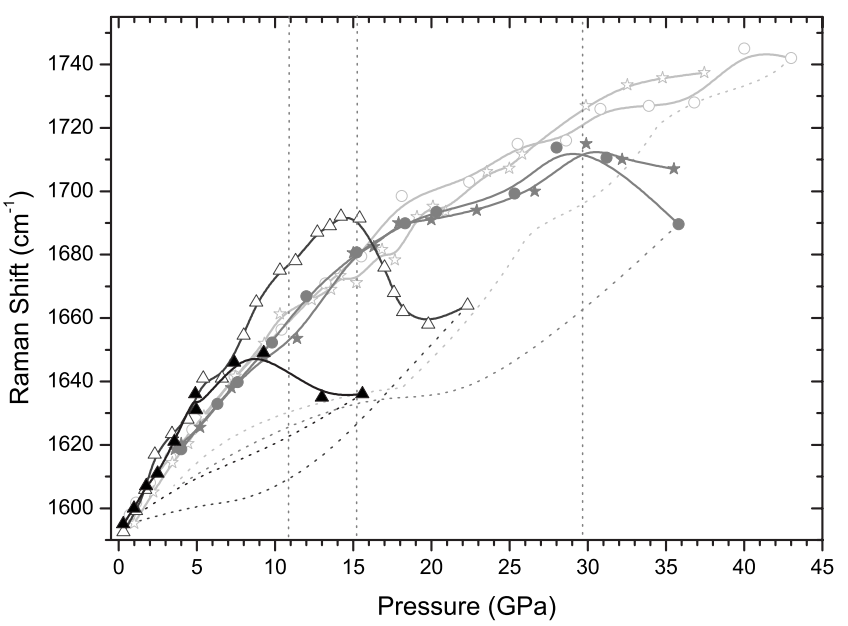

FIG. 7. Measured pressure evolution of the frequency of the TM maximum intensity for the peapods (filled symbols) and the empty nanotubes (hollow symbols) in oil (triangles), alcohol (circles), and argon (stars). The solid lines are guides for the eye for increasing pressures; the dashed lines represent the approximative paths during the decompression. The vertical dashed lines mark the transitions where the TM frequencies suddenly downshift. The data for empty nanotube in argon (hollow stars) are obtained from Ref. 23.

suggesting a strong interaction between the nanotube and the endohedral $\mathrm{C}_{70}$ in peapods. It is, however, interesting that the same type of TM downshift process seems to affect both the peapods and the empty nanotubes.

The most striking observation, which is the concomitant TM frequency downshift with the attenuation of the $\mathrm{C}_{70} \mathrm{Ra}-$ man modes, suggests the possibility that the nanotubes become strongly deformed. Nevertheless, the similar type of frequency downshift measured for peapods and nonfilled nanotubes excludes a physical origin of the observed downshift as due to the interaction between the tube and the fullerenes. Finally, the observation of a strong hysteresis also reinforces the idea that the TM frequency downshift should be related to important changes on the nanotube structure. For empty tubes, the flattening of the tube cross section [depicted in Fig. 6(c)], which would correspond to an important tube deformation compatible with our observations, has been predicted in this range of pressure. ${ }^{1,3}$ The TM frequency downshift can be explained by a change in the nanotube symmetry, as proposed in Ref. 41 , or/and by the interaction between the two sides of the nanotube wall. For peapods, as noticed previously, the flat shape is not expected for steric reasons. In fact, even if in intercalated systems the bulk modulus of fullerenes has been found to be smaller than in pristine phases, ${ }^{42}$ it remains exceptionally high and, for most purposes, fullerenes can be considered as nondeformable objects. For that reason, peapods may rather transform at high pressure toward the shape which was predicted in Ref. 43 at ambient conditions, in the case of peapods having small diameters. This type of deformation is depicted in Fig. 6(f). In such a case, the nanotubes are predicted to strongly interact with the encapsulated fullerenes, as expected here. Thus, the associated TM frequency downshift can be explained by similar processes: an alteration of the nanotube longitudinal 
symmetry or/and the interaction between the nanotube wall and the encapsulated $\mathrm{C}_{70}$ molecules. For both peapods and empty tubes, the strong hysteresis observed in Fig. 7 can be explained by a strong (attractive van der Waals bonding) interaction between the carbon layers, which would maintain the deformations during the release of the pressure. ${ }^{5}$

These different types of deformations may explain the lower transition pressure values obtained for peapods compared to empty nanotubes: $11 \pm 2 \mathrm{GPa}$ for peapods versus $14 \pm 1 \mathrm{GPa}$ for empty tubes in oil; $30 \pm 2 \mathrm{GPa}$ for peapods versus no transition up to $43 \mathrm{GPa}$ for empty tubes in alcohol or argon. It is interesting here to compare the different effects on the high pressure stability of SWNT depending on the type of tube filling, which we can qualify as disconnected in the case of fullerene filling (peapods) or connected in the case of filling with another carbon nanotube, i.e., in double wall carbon nanotubes (DWNTs). In fact, whereas in DWNT the inner tube supports the outer tube, leading to higher transition pressures, ${ }^{36,44,45}$ we have found that the opposite is observed in $\mathrm{C}_{70}$ peapods. The inhomogeneity in local constraints in peapods can be at the origin of such differences.

It is also worth noting that, contrarily to the first transition, the critical pressure of this second transition highly depends on the choice of PTM. PTM effects on the high pressure evolution of SWNT have already been reported. ${ }^{35,38,46-49}$ However, we note here that again alcohol and argon give exactly the same results both for peapods and nonfilled tubes. These "delayed" transitions, obtained when the PTM is alcohol or argon, may be explained by their higher hydrostaticity or the small size of the molecules, which may allow them to penetrate more easily in the bundle's interstitial regions and, thus, to stabilize the tubes. We can also consider the stabilizing effect of the penetration of the PTM inside the tubes as reported in Ref. 23.

In the case of nonfilled tubes, flattened structures have been predicted either as a first order phase transformation from the circular cross section with an important volume collapse ${ }^{5}$ or as a continuous evolution from an intermediate elliptic cross-section phase without volume collapse. ${ }^{1}$ Here, we first observe for the SWNT a low pressure transition corresponding to the change of the original circular cross section of the tubes [Fig. 6, (a) $\rightarrow($ b) $]$. This low pressure transition is found to be incompatible with a volume collapse (see Sec. III A) and is followed by a continuous and monotonous evolution of the Raman spectra (especially of the RBM enlargement and attenuation), which suggests a continuous change of the cross section. At higher pressure, the second transition is due to an important change of the nanotube structure. In correspondence with calculations, this second transition is attributed to the flattening of the tube's cross section in the case of nonfilled tubes [Fig. 6, (b) $\rightarrow$ (c)]. The predictions of Ref. 1 are probably those showing better agreement with our experimental results.

\section{Irreversible effects}

As can be seen in Figs. 2, 3, and 7, the two observed transitions appear as reversible and only minor modifications are found in the recovered Raman spectra. In the following,

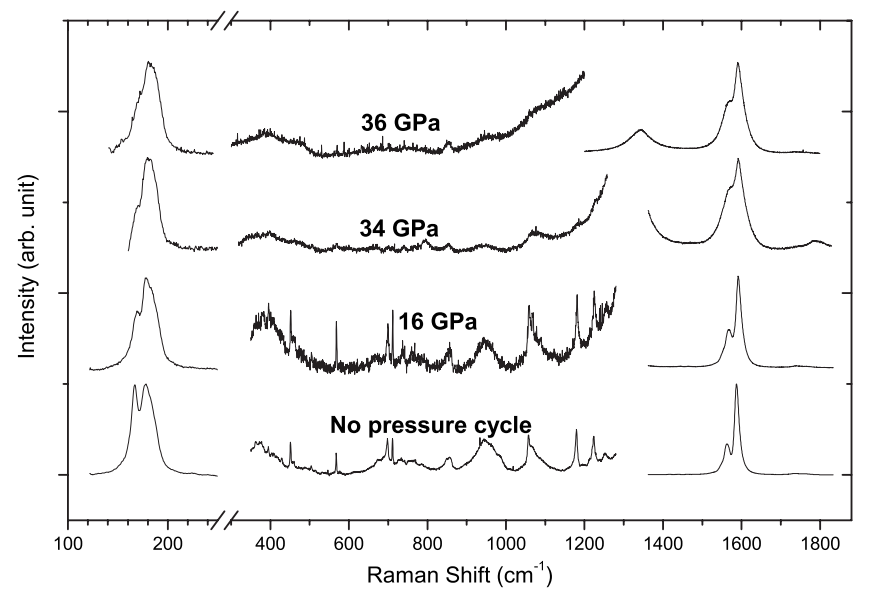

FIG. 8. Recovered spectra of peapods at ambient conditions after different pressure treatments $(16,34$, and $36 \mathrm{GPa})$ when the pressure transmitting medium is oil.

we discuss the presence of irreversible effects on peapods in the light of higher pressure treatments and TEM images.

In Fig. 8, we show the recovered Raman spectra of peapods at ambient conditions after different pressure treatments using oil as PTM. We observe with increasing pressure of treatment the rise of the intensity of the $D$ band, the attenuation of the RBM, and the enlargement of the TM. All these signs correspond to the creation of defects in the nanotubes. ${ }^{50}$ Interestingly, the attenuation of the RBM begins from low frequencies (see the mode around $165 \mathrm{~cm}^{-1}$ ), which correspond to the nanotubes of higher diameters. This is in agreement with the results obtained by photoluminescence in Ref. 17, suggesting that the structural damages are facilitated in nanotubes of large diameters. We observe a perfect reversibility of the frequencies of the nanotube intermediate modes and of those of $\mathrm{C}_{70}$, but also the attenuation of their intensity with increasing pressure. After a pressure treatment at $36 \mathrm{GPa}$, the $\mathrm{C}_{70}$ modes are not detectable, whereas a nanotube intermediate mode at $850 \mathrm{~cm}^{-1}$ is still observed. This attenuation is correlated to an intensity enhancement of the $D$ band.

In Fig. 9, we compare the recovered spectra of the peapods after a pressure cycle at $36 \mathrm{GPa}$ with the different PTMs. We observe exactly the same shapes for the RBM and the TM, but the $D$ band is more pronounced in the case of oil. The Raman signal of the $\mathrm{C}_{70}$ molecules has disappeared in the case of oil, whereas it is still present in the case of alcohol and argon. In these latter cases, the reversibility of the mode frequencies and relative intensities is perfect, except for the mode at $450 \mathrm{~cm}^{-1}$. This must be related to the PTM dependence found for the high pressure transition: once again, (i) the results are PTM dependent, (ii) alcohol and argon give the same results, and (iii) pressure effects seem to be delayed when alcohol or argon is used, compared to the case of oil.

In Fig. 10 are shown TEM images of the peapod bundles after pressure treatment at (a) $22 \mathrm{GPa}$ in oil and [(b)-(d)] $36 \mathrm{GPa}$ in alcohol. In Figs. 10(a) and 10(b), we observe that the length of the peapods is reduced with respect to the original sample (Fig. 1). This shortening of the peapod bundle 


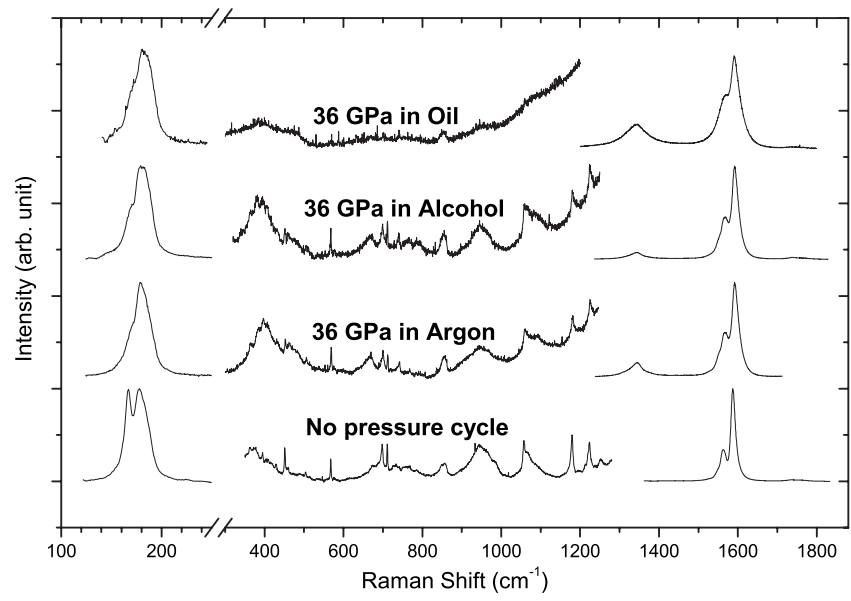

FIG. 9. Recovered spectra of peapods at ambient conditions after a pressure cycle up to $36 \mathrm{GPa}$ when using oil, alcohol, or argon as pressure transmitting medium.

length may be associated with shear forces related to the extrusion of the PTM after its solidification. We also observe in Figs. 10(c) and 10(d) the transformation of part of the original sample. In fact, in addition to the remaining peapods, we found additional nanostructures such as (c) multiwalled nanocones and (d) polygonized nano-onions, which were not detected by Raman spectroscopy. We can also observe in these two micrographs, close to these additional nanostructures, other structures that could correspond to disordered peapod bundles.

Finally, we see that high pressure treatments induce defects in the peapods (including their shortening), and can transform a part of them into other nanostructures. These nanostructures representing a mixture of multiwalled nanocones and disordered $s p^{2}$ clusters [see Fig. 10(c)] must contribute to the defects that are responsible for the increase of the $D$ band. It is worth noting that the $D$ band also rises when argon is used as PTM (Fig. 9). Therefore, these defects cannot solely be attributed to a chemical interaction between the nanotubes and the PTM, for instance, covalent bond creation. It is remarkable that the alteration of the peapods - and, thus, their transformation into other structures-begins from the nanotubes of larger diameters. This may be explained by the fact that the latter are more subjected to pressure-induced deformations.

After a pressure treatment at $36 \mathrm{GPa}$, contrarily to the case of alcohol and argon, the Raman spectra are strongly modified when the PTM is oil: the $\mathrm{C}_{70}$ modes disappear and the $D$ band is more intense. Moreover, the recovered sample was transformed into powder, and it was not possible to recover some of it for the TEM experiments. All this suggests that important transformations also occurred in this case. It is surprising to see that the (relatively) intense $\mathrm{C}_{70}$ modes disappear, while the nanotube intermediate modes are still visible. This looks like an alteration of the $\mathrm{C}_{70}$ molecules inside preserved nanotubes. Amorphization of pristine $\mathrm{C}_{70}$ have, indeed, been reported in the 20-35 GPa domain, with an irreversibility occurring presumably at $35 \mathrm{GPa} .{ }^{51,52}$ However, the creation of defects and the shortening of the tube can also
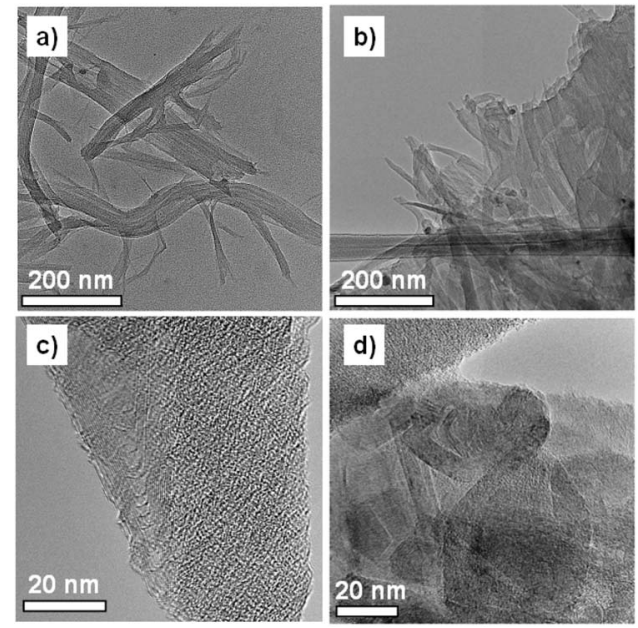

FIG. 10. Transmission electron microscopy images of the sample of peapods after pressure treatment. The nanotube bundles are shorter (broken) after a pressure treatment at (a) $22 \mathrm{GPa}$ in oil and (b) $36 \mathrm{GPa}$ in alcohol. Additional nanostructures are also formed after a $36 \mathrm{GPa}$ treatment in alcohol, like these (c) multiwalled nanocones and (d) polygonized nano-onions.

explain our observation. The nanotube intermediate modes have, indeed, been predicted to be tube-length dependent, with no intensity at all for infinite length. ${ }^{53}$ The relative intensity of the nanotube intermediate modes may have been enhanced by the shortening of the tubes. More generally, such a nanotube intermediate mode enhancement has been reported when defects are created in SWNT. ${ }^{54}$ Therefore, our observations are consistent with a very weak signal of the few untransformed (short and damaged) peapods, where only the nanotube modes can be seen.

\section{SUMMARY}

In situ high pressure studies monitored via Raman spectroscopy have been performed on bundles of single-walled carbon nanotubes filled with $\mathrm{C}_{70}$ molecules. We have provided strong evidence that the single wall carbon nanotubes undergo two transitions under pressure. These transitions are detected by a modification of the Raman spectra of the RBM and $\mathrm{TM}$, but also of the encapsulated $\mathrm{C}_{70}$ fullerenes when present. The first transition occurs at about $2-2.5 \mathrm{GPa}$ independent of the choice of the pressure transmitting medium, as well as of the filling or not of the tubes. The transition is characterized by the broadening of the RBM and is followed by a change in the slopes of the $\mathrm{C}_{70}$ Raman modes (when present) at $3.5 \mathrm{GPa}$. We explain this by a change of the nanotube cross section toward the predicted oval or polygonized shape, which causes the compression of the encapsulated fullerenes shortly after. We cannot exclude that this interaction is associated with the flip of standing $\mathrm{C}_{70}$ fullerenes toward lying configuration. The second transition takes place in the 10-30 GPa range. This transition highly depends on the choice of the PTM and the filling of the tubes. It is characterized by the simultaneous frequency downshift of the TM and disappearance of the $\mathrm{C}_{70}$ Raman modes (when 
present). We assign this behavior to the predicted flattening of the empty tubes and the deformation of the peapods toward a shape predicted for peapods of small diameters. In accordance with the literature, the observed TM frequency downshift can be due to the attracting van der Waals or bonding forces between the carbon layers (tube walls or fullerenes) or/and the alteration of the nanotube symmetry. Other possibilities such as the predicted tube interlink cannot be excluded. ${ }^{2}$ We have also evidenced the shortening of the tubes under pressure, the formation of additional nanostructures, and, in one case, the disappearance of the $\mathrm{C}_{70}$ Raman signal, either due to the alteration of the encapsulated fullerenes inside the tubes or the combined effect of transformations and defect creation in the tubes.

The two successive transitions experimentally observed here in single wall carbon nanotubes under pressure are in very good agreement with the theoretical predictions of Chan et $a l^{1}$ (bundled tubes) or Zang et al. ${ }^{3}$ (individual tubes). Nevertheless, they are in contradiction with some predictions of a sudden flattening transition occurring in the 1-2 GPa range for nanotubes in bundles. ${ }^{5}$ However, the effect of pressure may highly depend on the samples, especially between isolated and bundled tubes. For example, a negative compo- nent of the TM frequency, as is observed for our second transition, has also recently been observed in individualized empty tubes after a pressure transition around $2 \mathrm{GPa}$ by Freire et al. ${ }^{18}$ Therefore, further investigations may be necessary to determine on which conditions theoretical works and experiments can be in good agreement. We found a strong effect of the PTM on the higher pressure transition, which is the expression of the different interaction between the PTM molecules and the nanotubes. This can also be discussed in the light of our knowledge on nanocomposite materials, since the pressure transmitting media are solid at the considered pressures. The interface issues in nanofiber composites are well known as a key factor of the composite physical properties. We suggest that the different interphases obtained with the different PTMs are at the origin of our observations. This opens other perspectives for the study of the mechanical stability of carbon nanotube composite materials.

\section{ACKNOWLEDGMENT}

This work was supported by the Czech Ministry of Education through a grant LC-510.
${ }^{1}$ S.-P. Chan, W.-L. Yim, X. G. Gong, and Z.-F. Liu, Phys. Rev. B 68, 075404 (2003).

${ }^{2}$ S. Reich, C. Thomsen, and P. Ordejon, Phys. Status Solidi B 235, 354 (2003).

${ }^{3}$ J. Zang, A. Treibergs, Y. Han, and F. Liu, Phys. Rev. Lett. 92, 105501 (2004).

${ }^{4}$ R. B. Capaz, C. D. Spataru, P. Tangney, M. Cohen, and S. Louie, Phys. Status Solidi B 241, 3352 (2004).

${ }^{5}$ J. A. Elliott, J. K. W. Sandler, A. H. Windle, R. J. Young, and M. S. P. Shaffer, Phys. Rev. Lett. 92, 095501 (2004).

${ }^{6}$ M. Hasegawa and K. Nishidate, Phys. Rev. B 74, 115401 (2006).

${ }^{7}$ W. Yang, R. Z. Wang, X. M. Song, B. Wang, and H. Yan, Phys. Rev. B 75, 045425 (2007).

${ }^{8}$ A. N. Imtani and V. K. Jindal, Phys. Rev. B 76, 195447 (2007).

${ }^{9}$ U. D. Venkateswaran, A. M. Rao, E. Richter, M. Menon, A. Rinzler, R. E. Smalley, and P. C. Eklund, Phys. Rev. B 59, 10928 (1999).

${ }^{10}$ P. Teredesai, A. Sood, D. Muthu, R. Sen, A. Govindaraj, and C. Rao, Chem. Phys. Lett. 319, 296 (2000).

${ }^{11}$ M. J. Peters, L. E. McNeil, J. P. Lu, and D. Kahn, Phys. Rev. B 61, 5939 (2000).

${ }^{12}$ S. Rols, I. N. Gontcharenko, R. Almairac, J. L. Sauvajol, and I. Mirebeau, Phys. Rev. B 64, 153401 (2001).

${ }^{13}$ I. Loa, J. Raman Spectrosc. 34, 611 (2003).

${ }^{14}$ M. H. F. Sluiter and Y. Kawazoe, Phys. Rev. B 69, 224111 (2004).

${ }^{15}$ M. Lévy, J. Math. Pures Appl. 3, 5 (1884).

${ }^{16}$ J. Sandler, M. S. P. Shaffer, A. H. Windle, M. A. Montes-Morán, C. A. Cooper, R. J. Young, and M. P. Halsall, Phys. Rev. B 67, 035417 (2003).

${ }^{17}$ S. Lebedkin, K. Arnold, O. Kiowski, F. Hennrich, and M. M. Kappes, Phys. Rev. B 73, 094109 (2006).
${ }^{18}$ P. T. C. Freire, V. Lemos, J. A. Lima Jr., G. D. Saraiva, P. S. Pizani, R. O. Nascimento, N. M. P. S. Ricardo, J. Mendes Filho, and A. G. Souza Filho, Phys. Status Solidi B 244, 105 (2007).

${ }^{19}$ S. M. Sharma, S. Karmakar, S. K. Sikka, P. V. Teredesai, A. K. Sood, A. Govindaraj, and C. N. R. Rao, Phys. Rev. B 63, 205417 (2001)

${ }^{20}$ S. Klotz, J. M. Besson, G. Hamel, R. J. Nelmes, J. S. Loveday, W. G. Marshall, and R. M. Wilson, Appl. Phys. Lett. 66, 1735 (1995).

${ }^{21}$ M. H. Krisch, A. Mermet, A. San Miguel, F. Sette, C. Masciovecchio, G. Ruocco, and R. Verbeni, Phys. Rev. B 56, 8691 (1997).

${ }^{22}$ A. Souza-Filho, A. Jorio, G. G. Samsonidze, G. Dresselhaus, R. Saito, and M. S. Dresselhaus, Nanotechnology 14, 1130 (2003).

${ }^{23}$ A. Merlen, N. Bendiab, P. Toulemonde, A. Aouizerat, A. San Miguel, J.-L. Sauvajol, G. Montagnac, H. Cardon, and P. Petit, Phys. Rev. B 72, 035409 (2005).

${ }^{24}$ M. Chorro, J. Cambedouzou, A. Iwasiewicz-Wabnig, L. Noé, S. Rols, M. Monthioux, B. Sundqvist, and P. Launois, Europhys. Lett. 79, 56003 (2007).

${ }^{25}$ A. V. Soldatov, G. Roth, A. Dzyabchenko, D. Johnels, S. Lebedkin, C. Meingast, B. Sundqvist, M. Haluska, and H. Kuzmany, Science 293, 680 (2001).

${ }^{26} \mathrm{~S}$. Kawasaki, T. Hara, T. Yokomae, F. Okino, H. Touhara, H. Kataura, T. Watanuki, and Y. Ohishi, Chem. Phys. Lett. 418, 260 (2006)

${ }^{27}$ M. Chorro, S. Rols, J. Cambedouzou, L. Alvarez, R. Almairac, J.-L. Sauvajol, J.-L. Hodeau, L. Marques, M. Mezouar, and H. Kataura, Phys. Rev. B 74, 205425 (2006).

${ }^{28}$ Y. Zou et al., Phys. Rev. B 76, 195417 (2007).

${ }^{29}$ P. M. Rafailov, C. Thomsen, and H. Kataura, Phys. Rev. B 68, 193411 (2003). 
${ }^{30}$ H. Kataura, Y. Maniwa, M. Abe, A. Fujiwara, T. Kodama, K. Kikuchi, H. Imahori, Y. Misaki, S. Suzuki, and Y. Achiba, Appl. Phys. A: Mater. Sci. Process. 74, 349 (2002).

${ }^{31}$ L. Kavan, L. Dunsch, H. Kataura, A. Oshiyama, M. Otani, and S. Okada, J. Phys. Chem. B 107, 7666 (2003).

${ }^{32}$ A. Thess et al., Science 273, 483 (1996).

${ }^{33}$ A. Loiseau, P. Launois, P. Petit, S. Roche, and J.-P. Salvetat, Understanding Carbon Nanotubes, From Basics to Applications, Lecture Notes in Physics Vol. 677 (Springer-Verlag, Berlin, 2006).

${ }^{34}$ M. Chorro, A. Delhey, L. Noe, M. Monthioux, and P. Launois, Phys. Rev. B 75, 035416 (2007).

${ }^{35}$ A. Merlen, P. Toulemonde, N. Bendiab, A. Aouizerat, J.-L. Sauvajol, G. Montagnac, H. Cardon, P. Petit, and A. San Miguel, Phys. Status Solidi B 243, 690 (2006).

${ }^{36}$ J. Arvanitidis, D. Christofilos, K. Papagelis, K. S. Andrikopoulos, T. Takenobu, Y. Iwasa, H. Kataura, S. Ves, and G. A. Kourouklis, Phys. Rev. B 71, 125404 (2005).

${ }^{37}$ D. W. Snoke, Y. S. Raptis, and K. Syassen, Phys. Rev. B 45, 14419 (1992).

${ }^{38}$ P. Puech, H. Hubel, D. J. Dunstan, A. Bassil, R. Bacsa, A. Peigney, E. Flahaut, C. Laurent, and W. S. Bacsa, Phys. Status Solidi B 241, 3360 (2004).

${ }^{39}$ P. M. Rafailov and C. Thomsen, J. Optoelectron. Adv. Mater. 7, $461(2005)$

${ }^{40}$ L. Kavan and L. Dunsch, ChemPhysChem 8, 974 (2007).

${ }^{41}$ W. Yang, R.-Z. Wang, Y.-F. Wang, X.-M. Song, B. Wang, and H. Yan, Phys. Rev. B 76, 033402 (2007).

${ }^{42}$ R. Poloni, M. V. Fernandez-Serra, S. Le Floch, S. De Panfilis, P. Toulemonde, D. Machon, W. Crichton, S. Pascarelli, and A.
San-Miguel, Phys. Rev. B 77, 035429 (2008).

${ }^{43}$ M. Yoon, S. Berber, and D. Tomanek, Phys. Rev. B 71, 155406 (2005).

${ }^{44}$ P. Puech, H. Hubel, D. J. Dunstan, R. R. Bacsa, C. Laurent, and W. S. Bacsa, Phys. Rev. Lett. 93, 095506 (2004).

${ }^{45}$ V. Gadagkar, S. Saha, D. Muthu, P. K. Maiti, Y. Lansac, A. Jagota, A. Moravsky, R. Loutfy, and A. Sood, J. Nanosci. Nanotechnol. 7, 1753 (2007).

${ }^{46}$ S. Kawasaki, Y. Matsuoka, T. Yokomae, Y. Nojima, F. Okino, H. Touhara, and H. Kataura, Phys. Status Solidi B 241, 3512 (2004).

${ }^{47}$ P. Puech, E. Flahaut, A. Sapelkin, H. Hubel, D. J. Dunstan, G. Landa, and W. S. Bacsa, Phys. Rev. B 73, 233408 (2006).

${ }^{48}$ J. E. Proctor, M. P. Halsall, A. Ghandour, and D. J. Dunstan, J. Phys. Chem. Solids 67, 2468 (2006).

${ }^{49}$ M. J. Longhurst and N. Quirke, Phys. Rev. Lett. 98, 145503 (2007).

${ }^{50}$ M. Hulman, V. Skákalová, S. Roth, and H. Kuzmany, J. Appl. Phys. 98, 024311 (2005).

${ }^{51}$ N. Chandrabhas, A. K. Sood, D. V. S. Muthu, C. S. Sundar, A. Bharathi, Y. Hariharan, and C. N. R. Rao, Phys. Rev. Lett. 73, 3411 (1994).

${ }^{52}$ J. R. Patterson, S. A. Catledge, Y. K. Vohra, J. Akella, and S. T. Weir, Phys. Rev. Lett. 85, 5364 (2000).

${ }^{53}$ R. Saito, T. Takeya, T. Kimura, G. Dresselhaus, and M. S. Dresselhaus, Phys. Rev. B 59, 2388 (1999).

${ }^{54}$ V. Skákalová, J. Maultzsch, Z. Osváth, L. P. Biró, and S. Roth, Phys. Status Solidi (RRL) 1, 138 (2007). 